\title{
Numerical modelling of 3D reconnection
}

\section{Comparison between rotational and spinning footpoint motions}

\author{
I. De Moortel ${ }^{1}$ and K. Galsgaard ${ }^{2}$ \\ 1 School of Mathematics and Statistics, University of St Andrews, North Haugh, St Andrews, Fife KY16 9SS, Scotland \\ e-mail: ineke@mcs.st-and.ac.uk \\ 2 Niels Bohr Institute, Julie Maries vej 30, 2100 Copenhagen $\emptyset$, Denmark
}

Received 29 May 2006 / Accepted 21 July 2006

\section{ABSTRACT}

\begin{abstract}
The coronal magnetic field is constantly subjected to a variety of photospheric, footpoint motions, leading to the build up, and subsequent release, of magnetic energy. Two different types of footpoint motions are considered here, namely (large scale) rotating and (small scale) spinning, using 3D numerical MHD simulations. The initial model consists of two aligned, thin flux tubes, which are forced to interact due to the boundary driving of the footpoints. Two variations of this setup are studied, namely with and without an additional, constant, background magnetic field. The nature of the boundary motions determines the shape of the central current sheet, the driving force of the reconnection process, as well as the efficiency of the build up of quasi-separatrix layers (when $B_{\mathrm{bg}} \neq 0$ ). The reconnection process is more efficient for the rotating of the flux sources and when a background magnetic field is added. In general, heating due to large and small scale motions is of comparable magnitude when no background field is present. However, with an additional background magnetic field, heating due to small scale footpoint motions seems substantially more efficient.
\end{abstract}

Key words. magnetohydrodynamics (MHD) - Sun: corona - Sun: activity - Sun: magnetic fields

\section{Introduction}

Magnetic reconnection, driven by photospheric footpoint motions remains one of the most promising mechanisms to convert magnetic energy into heating the local plasma (see e.g. a recent review by Klimchuk 2006). Braiding of the footpoints causes a build up of free magnetic energy as well as the generation of current concentrations, allowing reconnection to take place (Parker 1972; van Ballegooijen 1986; Mikić et al. 1987; Galsgaard \& Nordlund 1996; Gudiksen \& Nordlund 2005). Developing the idea of footpoint braiding further, Priest et al. (2002) pointed out that simple footpoint motions are sufficient to form current concentrations both along separatrix surfaces (separating different connectivity regions) and separators (intersection of separatrix surfaces). Once strong enough, these current sheets will allow reconnection to occur, resulting in a re-arrangement of the coronal magnetic field structures and hence, altering the connectivity of the photospheric magnetic fragments. This continuous reconnection of the coronal magnetic field will lead to a significant contribution to the heating of the local coronal plasma. The ensemble of constant photospheric motions is likely to be one of the main drivers which maintains the $1 \mathrm{MK}$ background temperature of the Quiet Sun corona (see e.g. Parnell 2002; or Falconer et al. 2003). On the other hand, observational results presented by Pevtsov \& Acton (2001) suggest that the high-temperature corona $(>2 \mathrm{MK})$ is mostly associated with large-scale reconnection and the evolution of strong active region magnetic fields.

The coronal tectonics model of Priest et al. (2002) showed that elementary interactions between flux fragments are likely to be at least as important as energetic events due to emergence and cancellation of magnetic flux. Recently, several numerical simulations have modelled such simple footpoint motions, leading to elementary heating events, without relying on flux emergence or annihilation. For example, numerical simulations of flux tube tectonics by Mellor et al. (2005) confirm that simple lateral motions of a non-uniform magnetic field result in the rapid build up of current sheets. Similarly, the interaction between flux sources in a constant background magnetic field are investigated numerically by Galsgaard et al. (2000a), Parnell \& Galsgaard (2004) and Galsgaard \& Parnell (2005). Driven by the boundary motions, the flux systems are forced to interact, resulting in the two flux fragments connecting and subsequently disconnecting through separator reconnection (Haynes et al. 2006). Priest et al. (2005) use a reduced MHD model to compare the energy storage and heating at separators and separatrix surfaces. The energy dissipation at separators and separatrix surfaces is driven by (large scale) rotational and (small scale) spinning motions, respectively. These authors conclude that coronal heating is likely to be of comparable importance at separatrices and separators. In addition to separatrix surfaces and separators, quasiseparatrix layers (QSLs) (Priest \& Démoulin 1995; Démoulin et al. 1996) are also known to be sites of preferential current formation (Milano et al. 1999). However, the details of this process are still under investigation (Galsgaard \& Nordlund 1997; Galsgaard et al. 2000b; Titov et al. 2003; Galsgaard et al. 2003; Mellor et al. 2005; Démoulin 2006; Aulanier et al. 2005).

In this paper, we present 3D numerical simulations of the energy build up and subsequent release through magnetic reconnection, driven by spinning the footpoints of two adjacent flux tubes. These results are then compared to the result from De Moortel \& Galsgaard (2006, hereafter Paper I), which investigated the reconnection that occurs when the flux concentrations are rotated on the boundaries. The basic setup is described in Sect. 2, and to ease the comparison between the two different types of footpoint motions, a brief summary of the results from 


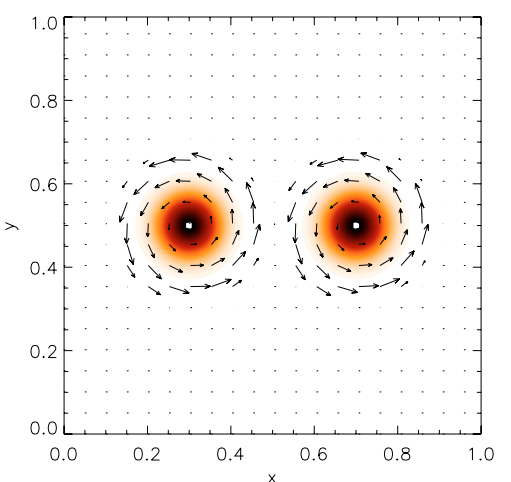

Fig. 1. Contour plot of the flux sources at the bottom boundary. The vectors indicate the "spin" driving velocity imposed on the bottom boundary of the numerical domain.

Paper I is given. The dynamical evolution of the spinning experiments is described in Sect. 3 and subsequently, the generation of current concentrations (Sect. 4), the connectivity structure and reconnection rates (Sect. 5) and the global energetics (Sect. 6) are investigated. A discussion of the results and the general conclusions can be found in Sects. 7 and 8, respectively.

\section{Numerical setup}

\subsection{Basic model}

The numerical domain contains the same basic setup as in Paper I, namely two aligned, thin flux tubes, which are modelled by considering two positive and two negative sources at the top $\left(T_{1}\right.$ and $\left.T_{2}\right)$ and bottom $\left(B_{1}\right.$ and $\left.B_{2}\right)$ of the cubical, numerical box (Fig. 1) with side length unity. The magnetic field distribution on the boundaries is given by

$B_{z}=B_{0}\left[\mathrm{e}^{-\left[\left(x-x_{1}\right)^{2}+\left(y-y_{1}\right)^{2}\right] / r_{0}^{2}}+\mathrm{e}^{-\left[\left(x-x_{2}\right)^{2}+\left(y-y_{2}\right)^{2}\right] / r_{0}^{2}}\right]+B_{\mathrm{bg}}$.

We choose $B_{0}=1$, the radius of the sources $r_{0}=0.065$ and the centre of the flux concentrations on both boundaries as $\left(x_{1}, y_{1}\right)=(0.3,0.5)$ and $\left(x_{2}, y_{2}\right)=(0.7,0.5)$. As the position of the sources is the same on the top and bottom boundary, the domain initially contains two straight magnetic flux tubes (see the first snapshot in Fig. 2). From the above source distributions on the boundaries, a potential magnetic field configuration is calculated to fill the interior of the data cube. The domain is assumed to be periodic in the horizontal directions. As in Paper I, two different variations of this setup are considered, namely with and without an additional, constant, magnetic background field. Throughout this present study, we have chosen this background field as $B_{\mathrm{bg}}=0.05$. The spinning driving profile considered in this paper is shown in Fig. 1 and is given by:

$$
\begin{aligned}
v(r)= & v_{0} r_{1}\left[1+\tanh \left(A\left(1-B r_{1}\right)\right)\right] \\
& +v_{0} r_{2}\left[1+\tanh \left(A\left(1-B r_{2}\right)\right)\right],
\end{aligned}
$$

where $r_{1}^{2}=\left(x-x_{1}\right)^{2}+\left(y-y_{1}\right)^{2}$ and $r_{2}^{2}=\left(x-x_{2}\right)^{2}+\left(y-y_{2}\right)^{2}$. The driving speed is taken as $v_{0}=0.02222$ and $A=16.8$ and $B=2.8$ determine the steepness and location of the drop off in $v$ outside the sources. Note that the velocity profile is designed to increase linearly with radial distance from the centre of each of the sources, in order to maintain the shape of the flux concentrations as they are spun. Again the motions on the top and bottom boundary are counter-rotated, with the anti-clockwise velocity vectors shown in Fig. 1 representing the spin on the bottom boundary. The angle (measured in radians) by which the sources (on one of the boundaries) have been rotated or spun will be used as a reference value to allow us to compare the two different types of footpoint driving.

When $B_{\mathrm{bg}}=0$, the numerical domain initially contains two separate flux domains, namely the vertical flux tubes (Fig. 2, top left) connecting $B_{1}$ to $T_{1}$ and $B_{2}$ to $T_{2}$, on either side of a central separatrix surface (the periodic boundaries represent independent sets of separatrix surfaces which are not important for the evolution of the central current sheet). However, when a positive background field is added $\left(B_{\mathrm{bg}}>0\right)$, there are no longer distinct flux domains, as the entire boundary now contains flux of the same polarity. In this case, we define a "flux tube" as the volume enclosed by the field lines traced from a radius, $r_{0}$, from the centre of the sources (the radius of the sources in the nobackground field experiment). Note that when such a positive background magnetic field is present, these flux tubes are not real topological features but merely a convenient notation to refer to the flux associated with each of the flux concentrations on the boundaries. Before the onset of the boundary driving, the flux tubes in the $B_{\mathrm{bg}}=0$ experiments are in contact, unlike in the $B_{\mathrm{bg}} \neq 0$ case, where the background field keeps the "flux tubes" separated.

To follow the dynamical evolution of the 3D system, the numerical code solves the non-ideal MHD equations in a 3D, Cartesian box, which is assumed to be periodic at the sides, and bounded in the vertical direction (i.e. the direction perpendicular to the plane in which the driver is situated). For a more detailed description of the numerical code, see Paper I and Nordlund \& Galsgaard (1997).

\subsection{Summary of rotational footpoint driving}

In the present paper, we want to compare the effect of two different types of footpoint motions on two adjacent flux tubes. The first type of boundary driving, the rotation of the flux concentrations around a common centre, is discussed in detail in Paper I. To ease the comparison with the results presented in the current study, we here briefly summarise the main conclusions of Paper I.

Due to the rotational motion of the flux concentrations on the boundaries, the straight magnetic flux tubes are deformed into two, interlinking, helical structures. The increasing tension in the rotated field lines results in a strong magnetic tension force, which initiates a stagnation point flow. As the shear in the magnetic field lines grows, the central current sheet (formed along the (quasi-)separatrix surface) gradually builds up and eventually becomes sufficiently strong to allow for the onset of reconnection. The presence of a background magnetic field delays the onset of reconnection between the two flux tubes, but once started, the reconnection process is more efficient for higher values of $B_{\mathrm{bg}}$.

\section{Dynamical evolution}

The spinning motion of the flux sources on the boundaries differs from the rotational boundary driving investigated in Paper I, in the sense that the position of the sources on the top and bottom boundaries remains unaltered and the spinning footpoint motions are localised, only affecting the flux concentrations themselves. The alignment of the flux sources stays the same at all times and hence, the spinning boundary driving has no effect on the potential field evolution; it will remain identical to the 

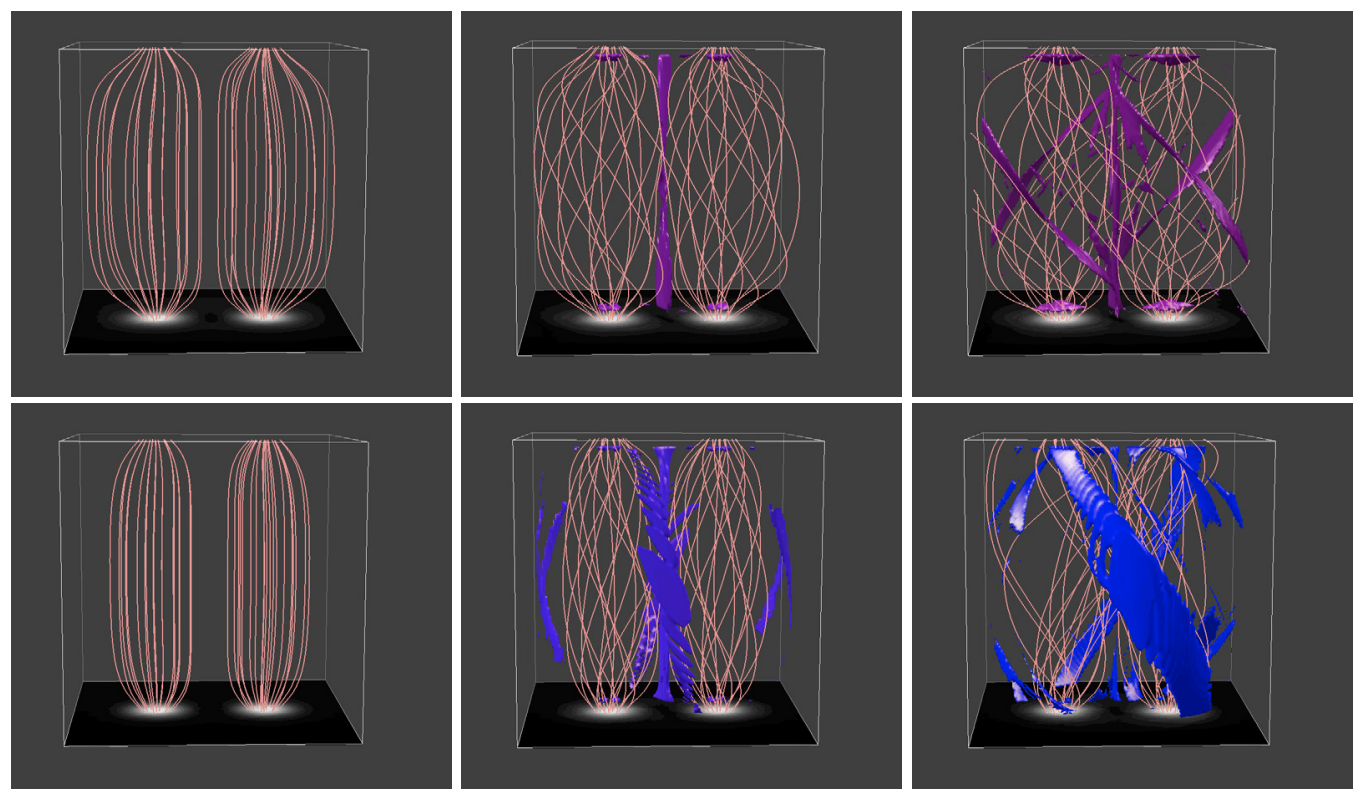

Fig. 2. Field lines traced from the flux concentrations on the bottom boundaries at a spinning angle (left) $\theta=0,($ middle) $\theta \approx 2$ and (right) $\theta \approx 4$ for $B_{\mathrm{bg}}=0$ (top) and $B_{\mathrm{bg}}=0.05$ (bottom). The isosurfaces represent current.

\section{Potential}

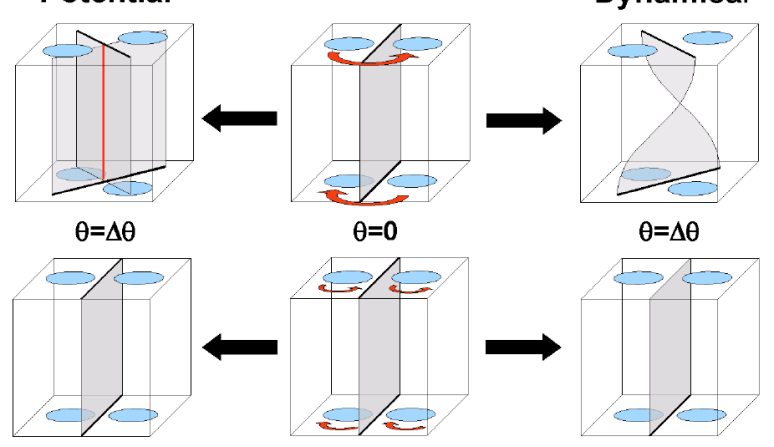

Fig. 3. Cartoon illustrating the difference in the potential and dynamical evolution of the topological domains for the (top) rotational and (bottom) spinning boundary driving.

initial setup, namely field lines connecting all the flux from $B_{1}$ to $T_{1}$ and $B_{2}$ to $T_{2}$. The rotational boundary motions on the other hand, caused a cyclic variation in the potential field evolution, as the alignment of the flux sources changed repeatedly.

Figure 2 shows three different stages of the dynamical evolution for both $B_{\mathrm{bg}}=0$ (top) and $B_{\mathrm{bg}}=0.05$ (bottom). Both sets of field lines are traced from the flux sources on the lower boundary and isosurfaces of strong current are also shown. (Note that the current near the boundaries of the numerical domain has been removed for clarity). The three snapshots correspond to spinning angles (left) $\theta=0$, (middle) $\theta \approx 2$ and (right) $\theta \approx 4$. As expected, the dynamical evolution is much more complicated than the potential one, with the field lines becoming increasingly twisted. Similar to the rotational case, a strong central current sheet builds up along the central (quasi-)separatrix surface, but rather than being twisted (see Fig. 2 in Paper I), it remains straight. This clearly shows the different nature of the two different types of footpoint motions, as illustrated by a cartoon in Fig. 3. At the start of the various experiments, the field lines going from the sources on the bottom boundary to the top sources are separated by a separatrix surface (separating the connectivity domains $B_{1}-T_{1}$ and $B_{2}-T_{2}$ ). The same is true for the field lines connecting the sources on the top boundary to the flux concentrations on the bottom boundary. Due to the symmetrical initial setup, these two separatrix surfaces coincide and when the sources are spun on the boundaries, this topological state remains unchanged until the onset of reconnection, regardless of whether one considers the potential or dynamical field evolution (see bottom row of Fig. 3). However, when the sources are rotated on the boundaries, there is a fundamental difference between the potential and dynamical field evolution. Due to the large scale rotational motions, the flux concentrations on the boundaries change position, hence altering their alignment. As reconnection is not instantaneous in the dynamical evolution, the central (coinciding) separatrix surfaces will become increasingly twisted. The potential field evolution, on the other hand, allows instantaneous reconnection and hence, the two central separatrix surfaces will rotate with the sources and intersect at a separator (top row in Fig. 3), dividing the numerical domain into four topological regions (when $B_{\mathrm{bg}}=0$ ). In this case, one could refer to the central current sheet as a "separator" or "separatrix" current sheet for the rotating and spinning boundary motions, respectively, as in Priest et al. (2005). This difference in the classification of the current sheets arises as these authors allow the field to reconnect instantaneously and thus form the two intersecting separatrices in the rotational experiment. This causes the formation of a separator line defined by the intersecting of the two separatrix surfaces (as seen in the top left frame of Fig. 3). As we focus our attention on the dynamical evolution, this splitting of the flux domains does not take place. In fact there is no difference in the nature of the central current sheet for the different types of footpoint motions, only in its shape: the large scale (rotational) motions generate a twisted current sheet, whereas the small scale (spinning) motions lead to the build up of a straight current sheet. As such, we will not use the distinction between separator and separatrix current sheet as in Priest et al. (2005), but simply refer to the central current sheet.

As the spinning of the sources on the boundaries continues, the field lines become increasingly twisted and the central current sheet eventually becomes strong enough to allow 

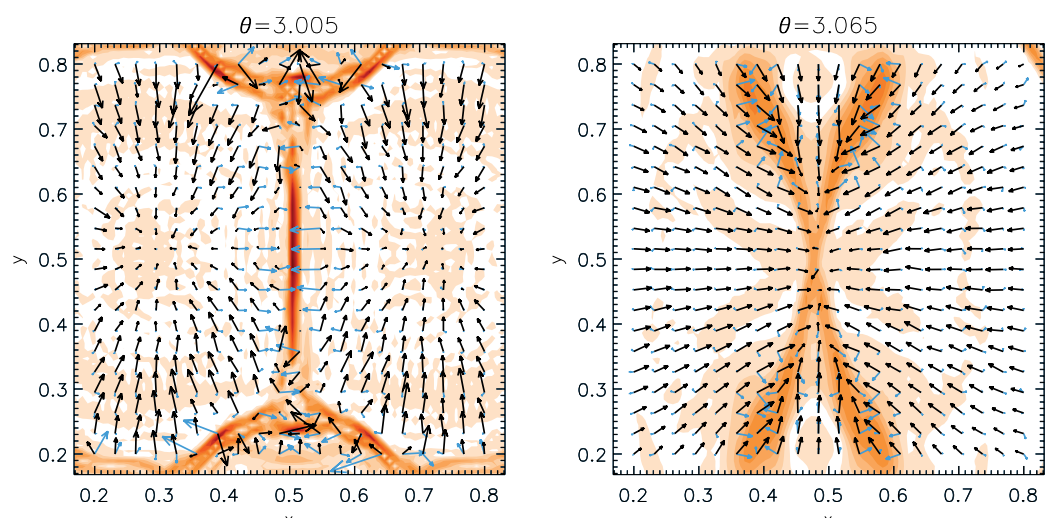

Fig. 4. Contours of current at $z=0.5$, with $\theta \approx 3$, for both the (left) spinning and (right) rotating boundary motions, where $B_{\mathrm{bg}}=0$. Over plotted are cotemporal vectors of the gradient of the magnetic pressure (blue) and the tension force (black).

Table 1. An overview of the spinning angles at which various stages of the reconnection occur, for different values of the additional background magnetic field, $B_{\mathrm{bg}}$.

\begin{tabular}{lcc}
\hline \hline & $B_{\mathrm{bg}}=0$ & $B_{\mathrm{bg}}=0.05$ \\
\hline Onset of reconnection & 1.46 & 1.86 \\
25\% reconnected flux & 3.44 & 2.87 \\
$50 \%$ reconnected flux & 4.93 & 3.53 \\
75\% reconnected flux & - & 4.25 \\
100\% reconnected flux & - & 5.20 \\
End of experiment & 6.87 & 6.49 \\
\hline
\end{tabular}

reconnection to take place. In the $B_{\mathrm{bg}} \neq 0$ case, the background field has a confining effect on the two initial flux tubes and initially keeps them separated. This background field has to be removed before reconnection between the flux tubes is possible. As the sources are spun, magnetic pressure builds up, leading to a radial expansion of the flux tubes. This causes field lines from the two flux tubes to collide and results in the build up a strong central current sheet. Figure 4 shows contours of the current at $z=0.5$, with $\theta \approx 3$, for both the (left) spinning and (right) rotating boundary motions (where darker colours correspond to higher values). Over plotted are cotemporal vectors of the gradient of the magnetic pressure (blue) and the tension force (black). The slight asymmetry in the vectors is due to the fact that the current sheet does not align with the centre of the grid cells. For the spinning of the flux concentrations (Fig. 4, left), we can see that it is indeed the magnetic pressure (blue arrows) that carries the field lines towards the strong central current sheet. In the rotating case (Fig. 4, right), on the other hand, it is mainly the magnetic tension (black arrows) in the rotated field which drives the field lines toward the central reconnection region. As reconnection takes place, new flux domains are created $\left(B_{1}\right.$ to $T_{2}$ and vice versa) and the footpoint connectivity gradually changes. The additional flux domains are created in both the $B_{\mathrm{bg}}=0$ and $B_{\mathrm{bg}}=0.05$ experiments, with a QSL forming between the two flux tubes in the $B_{\mathrm{bg}} \neq 0$ case. We will describe the evolution of the QSL's in more detail in Sect. 4. The right hand side snapshots in Fig. 2 show that at the later stages of the evolution, many current fragments are present throughout the domain, which are evidence of the many different interactions between the field coming from the sources and the background field, as well as interactions through the periodic side-boundaries of the computational domain.

Table 1 provides an overview of the time at which a given amount of flux has reconnected, as a percentage of the total flux of a source. As indicated above, the onset of reconnection between the two flux tubes occurs later (i.e. at a larger value of the spinning angle $\theta$ ) when a background magnetic field is present, due to its confining effect on the flux tubes. However, once started, the amount of reconnected flux increases much faster in the experiment where a background field has been added. A similar result was found in Paper I for the rotational driving. In Sect. 5 we will make a more detailed comparison between the rate of change of the footpoint connectivity of the various experiments.

\section{Current evolution}

As pointed out in the previous section, the different types of boundary motions lead to differences in the central current sheet. To investigate the nature of the current sheet in the spinning experiments in more detail, we focus on the current structures in the $z=0.5$ plane. Figure 5 shows a series of snapshots of contours of current, at mid-height in the computational box, for (top) $B_{\mathrm{bg}}=0$ and (bottom) $B_{\mathrm{bg}}=0.05$. Lighter colours represent higher values of current. In the initial phase of the boundary driving, the current structure in the $B_{\mathrm{bg}}=0$ and $B_{\mathrm{bg}}=0.05 \mathrm{ex}-$ periments looks quite similar: current builds up at the edges of the "flux tubes" due to the shear caused by the strong decrease in the amplitude of the driving velocity (see Fig. 1). These two circles of current meet in the middle of the domain, resulting in a strong central current sheet, caused by the shearing between the two flux tubes (in the $B_{\mathrm{bg}}=0$ case) or between the background field (in the $B_{\mathrm{bg}} \neq 0$ case). For the no-background field experiment, this strong current sheet remains present at the centre of the domain throughout the simulation.

As reconnection starts, two extra flux domains are created, and in e.g. the second snapshot in Fig. 5, we can now clearly see the four different domains: left and right of the current sheet are the two original flux tubes $\left(B_{1}-T_{1}\right.$ and $\left.B_{2}-T_{2}\right)$ and above and below the central current sheet we can see the newly formed domains $\left(B_{1}-T_{2}\right.$ and $\left.B_{2}-T_{1}\right)$, containing the reconnected field. As the boundary driving continues (snapshots from left to right), the original flux domains become smaller while simultaneously, the domains with reconnected field extend in size. The four different domains are shown clearly in the snapshot at $\theta=2.36$ in Fig. 8 (top), where the original connections are shown in yellow and blue, and the reconnected field is drawn in green and red. In the final stages of the evolution, there are many fragments of strong current throughout the computational domain (see also Fig. 2, right), caused by the interactions between the 

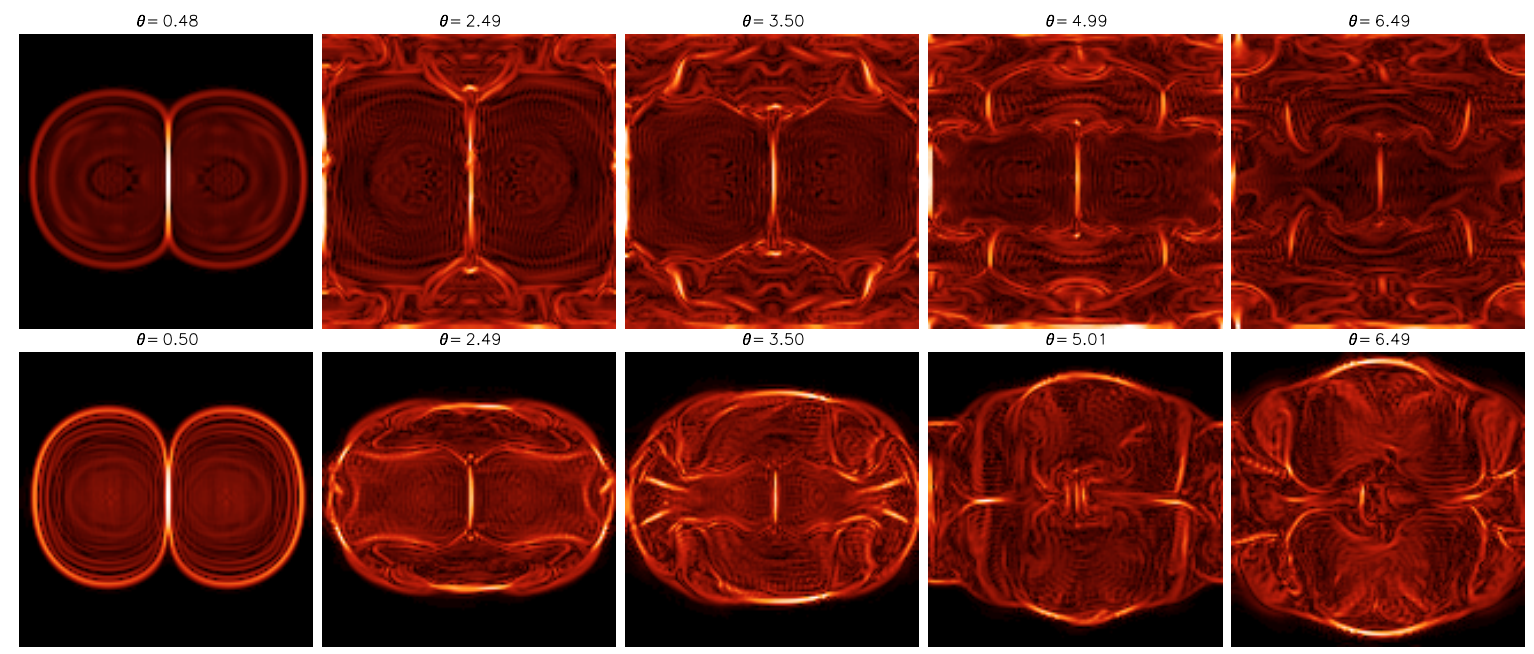

Fig. 5. Contour plots of the current at $z=0.5$ at various different spinning angles, for the case without (top) and with (bottom) background field, $B_{\text {bg }}=0.05$.
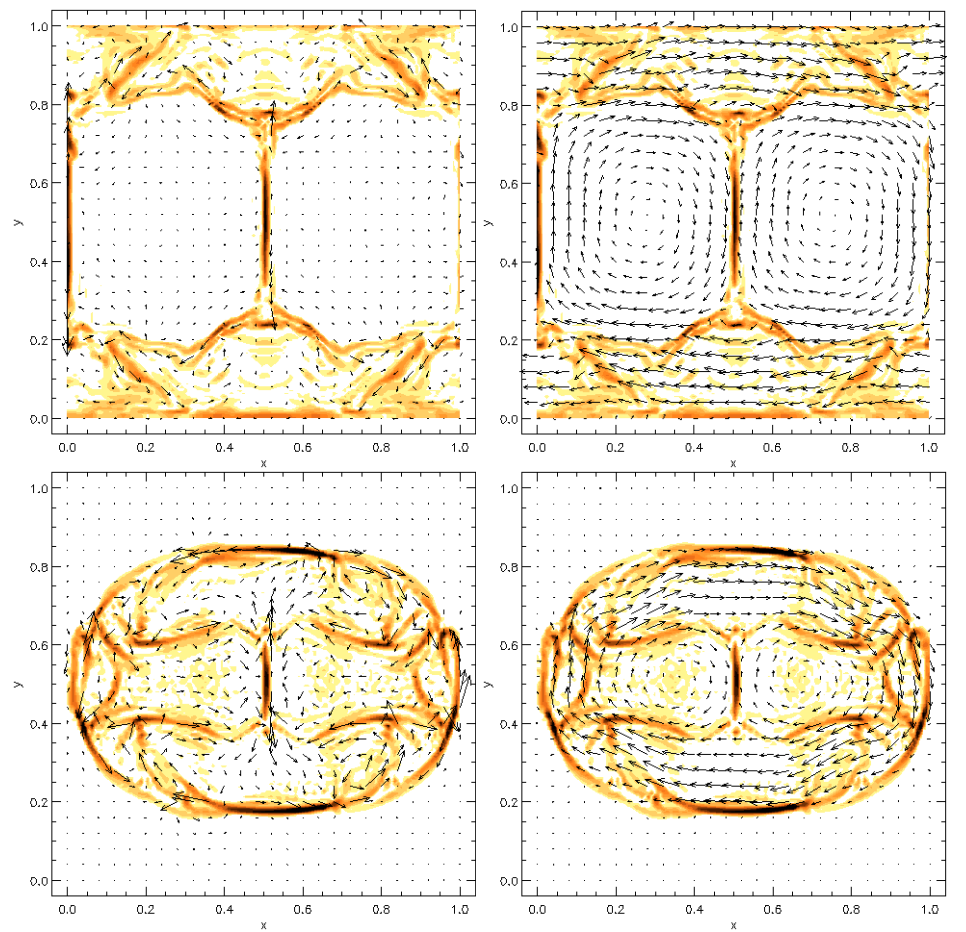

Fig. 6. Contour of the current at $z=0.5$ and $\theta \approx 3$ for the case without (top) and with (bottom) background field $\left(B_{\mathrm{bg}}=0.05\right)$. Over plotted are the cotemporal velocity (left) and magnetic field (right) vectors in the $z=0.5$ plane.

flux tubes and the reconnected field, the background field (when present) and the periodic boundaries of the box. When a background field is present (Fig. 5, bottom row), the evolution of the different (quasi-)domains is largely similar to the no-background case, apart from the fact that the entire current structure is limited in size due to the confining effect of the background field. Note, however, how this entire region expands and at the end of the experiment, almost fills the complete domain. Also in these final stages, the central current sheet has completely disappeared for the $B_{\mathrm{bg}}=0.05$ experiment, as $100 \%$ of the flux has changed connectivity for $\theta>5.20$.

Figure 6 shows the contours of the current at $\theta \approx 3$, with cotemporal velocity (left) and magnetic field (right) vectors over plotted, both for $B_{\mathrm{bg}}=0$ (top) and $B_{\mathrm{bg}}=0.05$ (bottom). Focusing on the magnetic field first confirms that the strong current regions are located in regions of strong shear in the magnetic field, for both experiments. At this stage (see Table 1), the reconnection process is well underway and looking at the velocity vectors, we can see that there is a (relatively weak) flow carrying field towards the central current sheet. Unlike the rotational case, where a stagnation point flow was initiated by the tension in the rotated field lines, the flow in this case is the result of a build up in magnetic pressure. The outflow velocities along the central current sheet are much larger in magnitude than the inflow velocities, carrying field rapidly away, into the "reconnected" domains, which are here clearly visible above and below the current sheet. These current contours also show that the current has a "top-hat" profile along the current sheet, i.e. the magnitude of the current inside the sheet is fairly constant along the line $x=0.5$. 

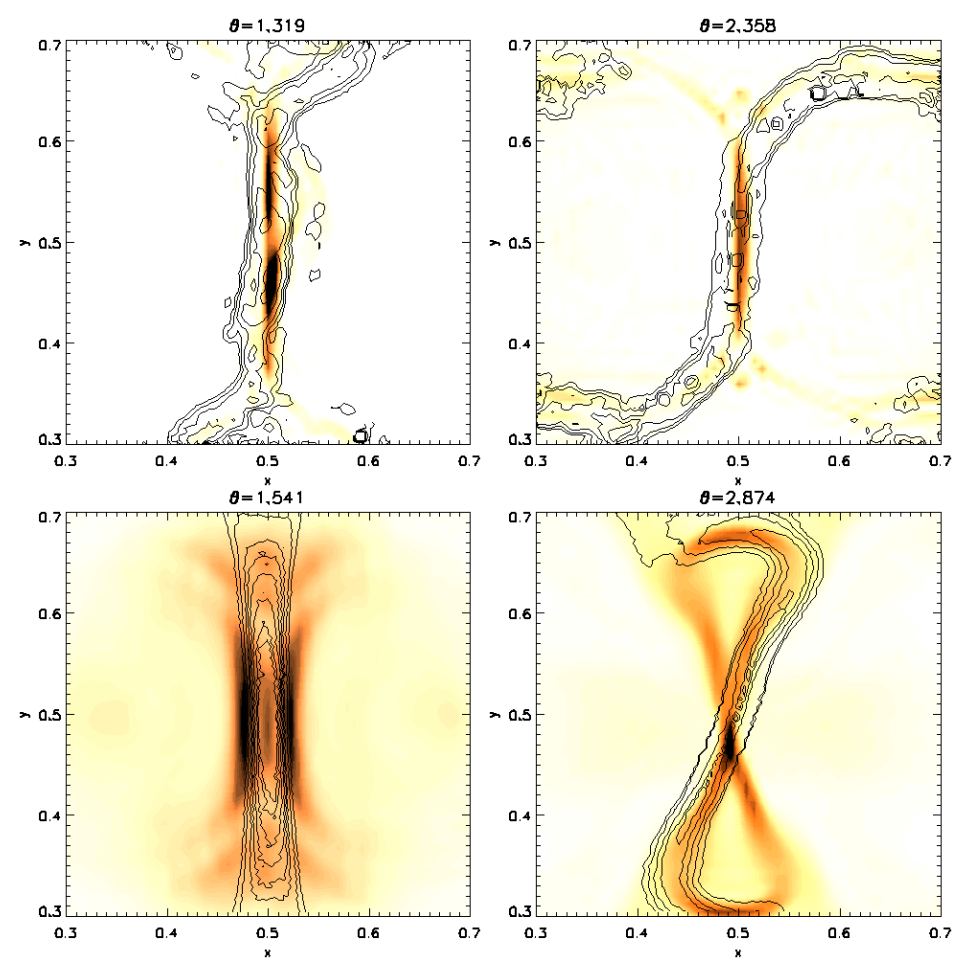

Fig. 7. Contour plots of the current at $z=0.5$ (central square of width 0.4 ) for $B_{\mathrm{bg}}=0.05$ at different spinning (top) and rotating (bottom) angles. The black line contours correspond to high values of the squashing factor $Q$.

When a background magnetic field is present, there is a continuous transition between the regions of different connectivity and hence, there are no real, distinctly separate flux domains. In this case, an elegant way of identifying regions with a rapid (rather than discontinuous) change in connectivity is evaluating the degree of squashing, $Q$ : regions of high $Q$-value are known as quasi-separatrix layers (QSL's), where the requirement of a discontinuous jump in connectivity has been weakened to a "large" change in connectivity (Priest \& Démoulin 1995; Titov et al. 2002). In Fig. 7, contours of current in the $z=0.5$ plane are again plotted for different values of the (top) spinning and (bottom) rotational angle. Over plotted are contour lines corresponding to high values of the squashing factor $Q$. Regardless of the spinning or rotating angle and regardless of the type of boundary motions, it is clear that currents build up in regions of strong shear in magnetic connectivity. Only part of the structure is visible in the $Q$-values in Fig. 7, as the tracing of the field lines, on which the determination of $Q$ is based, was only done in one direction (from the mid-plane to the top boundary).

The graphs in Fig. 7 (left) show the structure of the central current sheet at an early phase of the experiment (spinning: $\theta=1.32$ - rotating: $\theta=1.54$ ), i.e. before the onset of reconnection between the flux tubes. At this stage, we have a straight, relatively narrow, central current sheet for the spinning case, and a correspondingly narrow QSL. Early on in the rotational experiment however, the QSL is relatively broad. The figures on the right show the change in the central current sheet after the onset of reconnection (spinning: $\theta=2.36$ - rotating: $\theta=2.87$ ) and there is now a clear difference between the spinning and rotating experiments: the current sheet has bifurcated into a real $x$-type configuration intersecting at a single point for the rotational driving. For the spinning of the flux concentrations, the current takes on the shape of an "elongated" $x$-structure.

Let us now focus in more detail on these differences between the rotational and spinning boundary driving. In Paper I, we saw that the rotation of the flux concentrations leads to two, interlinking flux tubes, with a strong tension in the field lines, generating a stagnation point flow towards a relatively small, central reconnection region. When the flux sources are spun rather than rotated, this strong tension force does not build up and instead, the field lines are driven towards the central regions by the increasing magnetic pressure. However, rather than acting towards a single central region like the (rotational) tension, this increased magnetic pressure results in a general expansion of the flux tubes and thus, the pressure is (relatively) higher in the inflow regions than in the outflow regions, which get compressed. Hence, the central current sheet does not show a traditional, clear $x$-type structure for the spinning of the footpoints but has been squashed to resemble two $y$-points connected by the central current structure - i.e. this structure resembles the collapsed $x$-point as described by Green (1965) and Syrovatskii (1981). This difference in the central current structures is clearly visible in Fig. 7.

Although the general, dynamical evolution is similar for both types of boundary motions, the driving force of the reconnection process (as described in the previous paragraph) and the spatial extent of the reconnection region are quite different. Figure 8 shows a top and side view of the numerical domain for both the (top) spinning and (bottom) rotating of the flux concentrations. Field lines still at their original connections are shown in yellow and blue, whereas reconnected field is shown in green and red. Also shown are contours of current in the $z=0.5$ plane. Firstly, comparing the top and side view again confirms that regions of strong current coincide with a rapid change in the orientation of the magnetic field lines, or regions of different magnetic connectivity. This comparison also shows how, in this 3D configuration, field lines can appear parallel from one line of sight, but strongly sheared when viewed from a different angle.

From looking at the top row of Fig. 8, it can be seen that the extended width of the current sheet is the result of the compression of the central region between the two spun flux tubes. 

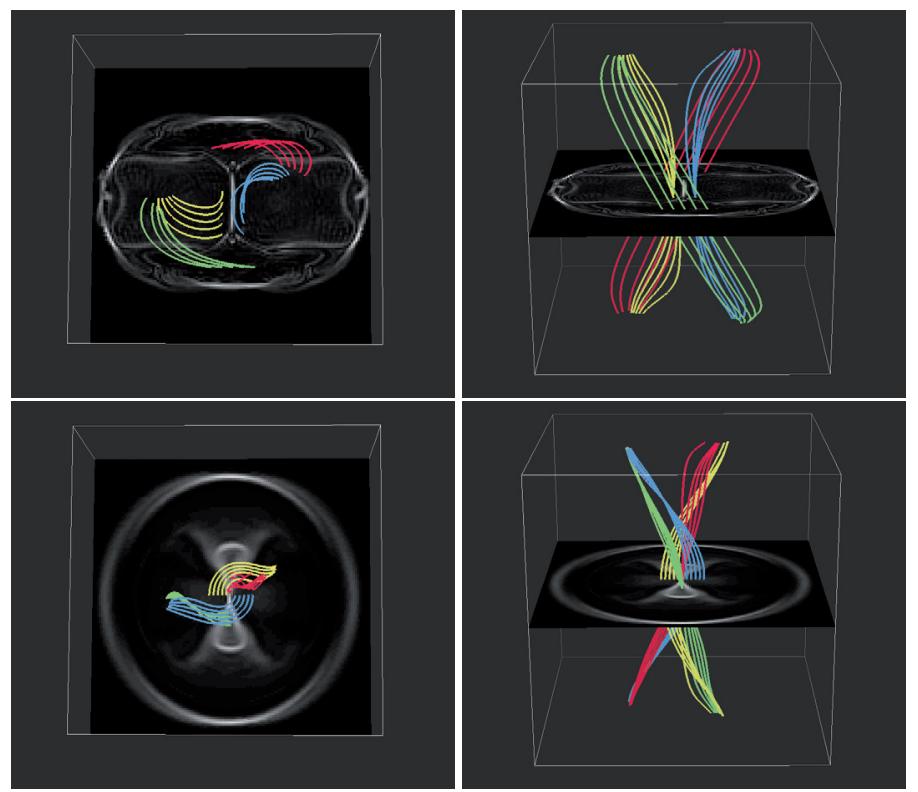

Fig. 8. The field line connectivity (viewed from above and the side) of the $B_{\mathrm{bg}}=0.05$ case for (top) the spinning driver at $\theta \approx 2.36$ and (bottom) the rotating driver at $\theta \approx 2.87$. The yellow and blue field lines represent the original connectivity, while the red and green field lines have reconnected. The contours represent the current at $z=0.5$.

In this case, the reconnected field lines are forced out along the current sheet, forming a small topological region. The current structures at the edge of these regions show a very large opening angle, representing the border between the different regions of flux connectivity. In comparison, the rotating experiment forms a very narrow, localised current sheet, which in form resembles a 2D Petschek-reconnection scenario, with a narrow current sheet and large extended slow mode shocks (Petschek 1964). The reason for the narrow current sheet is to be found in the twisting of the field lines, generated by the specific nature of the boundary motions; due to the rotating of the flux regions around one another, it is only possible for the current sheet to reach a limited extent as it is constantly being squeezed by the flux structures connecting the $B_{1}$ to $T_{1}$ and $B_{2}$ to $T_{2}$ flux regions. The different structure of the topological domains is also outlined by the structure of the QSL's in Fig. 7. Keeping in mind that in these figures only half of the QSL's are visible, the same $x$-type and elongated $x$-structure can be inferred for the rotational and spinning boundary motions, respectively.

Finally, we compare the build up of the maximum current and $Q$-value for the four different experiments. Figure 9 (top) shows the evolution of the maximum current density in the central square of width 0.4 at $z=0.5$ as a function of the angle $\theta$, where the thick and thin lines correspond to the $B_{\mathrm{bg}}=0$ and $B_{\mathrm{bg}}=0.05$ case, respectively. Solid lines represent the spinning driver, the dashed curves the rotational driver. It is immediately obvious that the maximum current builds up much faster (i.e. at smaller angles) and (initially) to higher values for the spinning boundary motions, despite the fact that these boundary motions affect a much smaller part of the magnetic domain and hence, less Poynting flux is injected into the domain (see Sect. 6 for a more detailed description of the energetics). However, looking at the nature of the boundary driving (see Fig. 1) shows that the spinning of the flux concentrations results in a very strong shear in the centre of the domain and hence, a rapid build up of the current. When the field is rotated, the driver does not affect the central regions as much (see Fig. 1 in Paper I), hence the slower build up of the central current sheet.

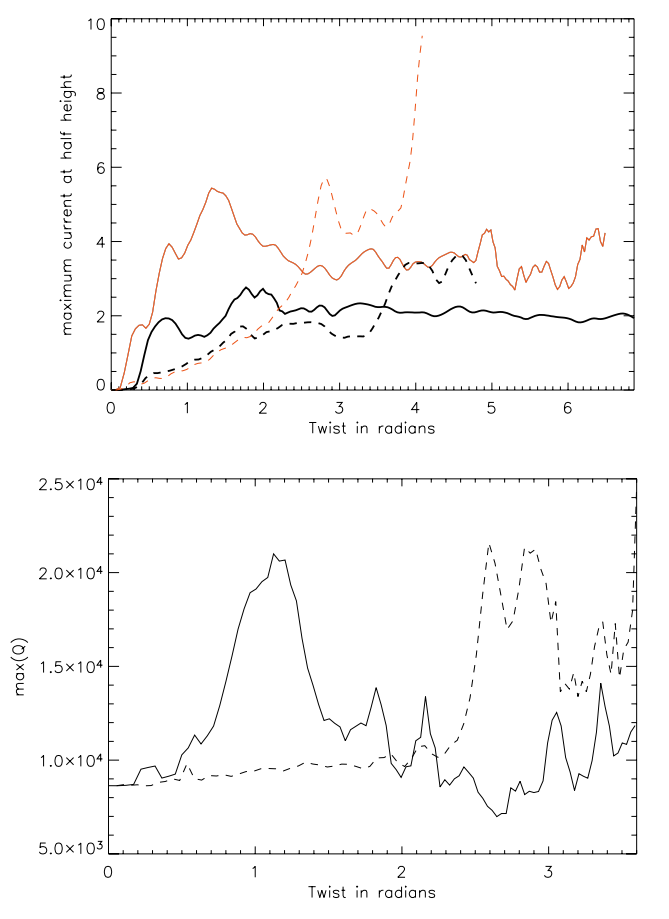

Fig. 9. (top) The maximum current, in the central square of width 0.4 at $z=0.5$, as a function of the angle $\theta$. The thick lines represent the $B_{\mathrm{bg}}=0$ cases, the thin lines $B_{\mathrm{bg}}=0.05$. Solid lines correspond to the spinning driver, the dashed ones to the rotational driver. (bottom) Similar to the graph on the right but for the maximum $Q$-value (and only for $B_{\mathrm{bg}}=0.05$ ).

However, as the rotating boundary motions continue, the field lines associated with the flux tubes take on an increasingly helical shape. When they finally enter the central region (after the background field has been removed), there is a large shear between the field lines associated with the two flux tubes, resulting in a sudden, very rapid growth of the maximum current density. 

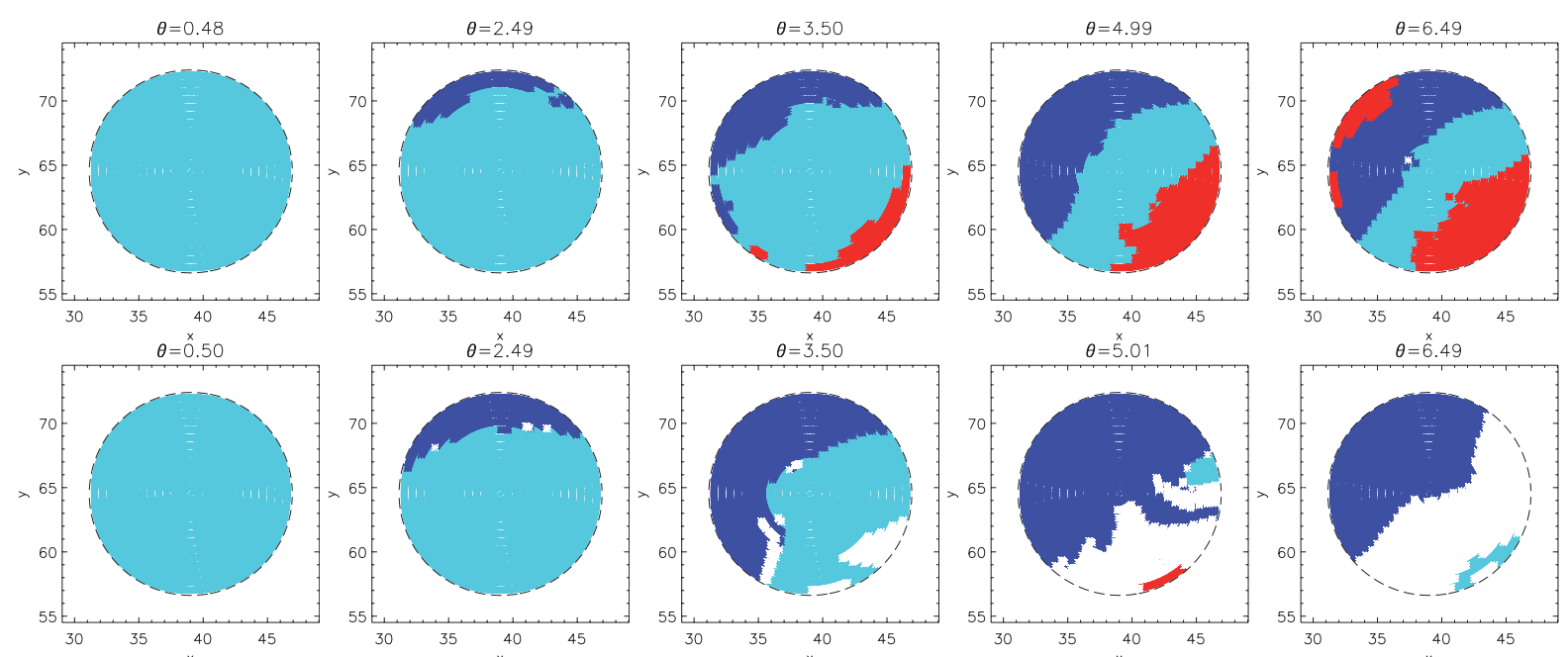

Fig. 10. The field line connectivity of one of the sources on the lower boundary, at different spinning angles, for the dynamical evolution with $B_{\mathrm{bg}}=0$ (top row) and $B_{\mathrm{bg}}=0.05$ (bottom row). The pale blue colour corresponds to field lines at their original connection, the dark blue shows field lines that have reconnected to the other flux source whereas the red corresponds to field lines that are leaving through the sides of the box and white are field lines connected to parts of the top boundary not associated with the flux concentrations.

The rapid build up of shear in the central region is reflected in the strong growth of the $Q$-value in the spinning experiment (Fig. 9, bottom), very soon after the onset of the boundary driving. Similar to the current density, the $Q$-value in the central region builds up later for the rotational driving of the flux sources.

\section{Connectivity and reconnection rates}

The evolution of the connectivity is studied in the same manner as in Paper I, namely following the method of Parnell \& Galsgaard (2004): a large number of field lines is traced from the sources on the bottom boundary at every time step, which allows us to calculate the amount of reconnected flux, assuming the flux is frozen into the plasma. Figure 10 shows a series of snapshots for both (top) the $B_{\mathrm{bg}}=0$ and (bottom) the $B_{\mathrm{bg}}=0.05$ case: field lines have been traced from within a radius $r_{0}$ of the centre of one of the flux concentrations on the bottom boundary; if the traced field lines end up within a radius $2 r_{0}$ from the centre of a source on the top boundary, we assume that they are connected to that source. The footpoints of the field lines which remain at their original connection are coloured pale blue, whereas the ones that have reconnected to the other source on the top boundary are dark blue. Field lines that are leaving through the (periodic) side boundaries of the computational domain are shown in red and finally, field lines that connect to parts of the top boundary not associated with the flux concentrations (i.e. outside a radius of $2 r_{0}$ from the centre of both sources) are represented in white.

As described in Sect. 3, the potential evolution is of little interest when the flux concentrations are spun on the boundaries; as the alignment of the flux sources on top and bottom boundary remains the same, the connectivity of the field lines will remain the same at all times. In terms of Fig. 10, the entire footpoint would remain pale blue during the corresponding potential evolution. It is obvious that the dynamical evolution does show a change in the footpoint connectivity, regardless of the presence of a background magnetic field. Let us firstly focus on the top row, i.e. on the $B_{\mathrm{bg}}=0$ experiment. Those field lines that are closest to the central current sheet, along the entire width of the current sheet will reconnect first. Due to the symmetrical nature of the experiment, we expect that to be those field lines that are closest to the location of the current sheet at different heights between the two driving boundaries. In the $z=0.5$ plane, these field lines will be the ones that were initially "facing" the other flux tube, due to the anti-symmetric driving. The driving on the two boundaries will make their footpoints counter-rotate and at the time they reconnect, they will be registered to belong to a particular region on the boundaries, namely the region that has rotated with a given angle, corresponding to the time at which reconnection occurs. Hence, if the onset of reconnection occurs at a spinning angle $\theta_{\mathrm{s}}$, the footpoints of the first field lines to reconnect will be at the outer edge of the (left-hand-side) source and rotated (anti-clockwise) roughly an angle $\theta_{\mathrm{s}}$ away from the right hand edge of the source (i.e. the point which at $t=0$ faced the other flux tube). For the $B_{\mathrm{bg}}=0$ case, the reconnection-onset angle is $\theta_{\mathrm{s}}=1.46$ and we see that the first cluster of reconnected (blue) field lines does indeed occur near the top of the source, i.e. roughly spun 90 degrees from the right hand edge of the source. The reason for the spreading of the region that experiences a connectivity change along the outer edge of the source arises from the reconnection taking place along a larger extent of the height ( $z$ direction) of the current sheet. As the sources are spun further, the amount of reconnected flux increases, with the "dark blue" area (i.e. the reconnected flux) extending along the edge of the source and towards the centre. Obviously, as the boundary driving continues, this reconnected fraction not only grows, but continues to be spun anti-clockwise. After a spin of about $\theta \approx 3$, field lines (red) also start to reconnect through the periodic side boundaries of the computational domain. The field lines at the left hand side of the source were originally closest to the boundary of the box and indeed, the red area is situated more or less directly opposite from the dark blue area.

When a background field is present, the evolution of the footpoint connectivity is largely similar to the one described above for the no-background experiment. Although the reconnection between the two flux tubes starts slightly later, the amount of reconnected (dark blue) flux increases faster than in the $B_{\mathrm{bg}}=0$ case. In the earlier stages of the experiment most of the reconnected field lines connect to the other source, rather than the background magnetic field. However, in the final stages of the experiment, a large amount of (white) field lines have connected to parts of the top boundary associated with the background field (i.e. outside the $2 r_{0}$ radius around both sources). Note also 

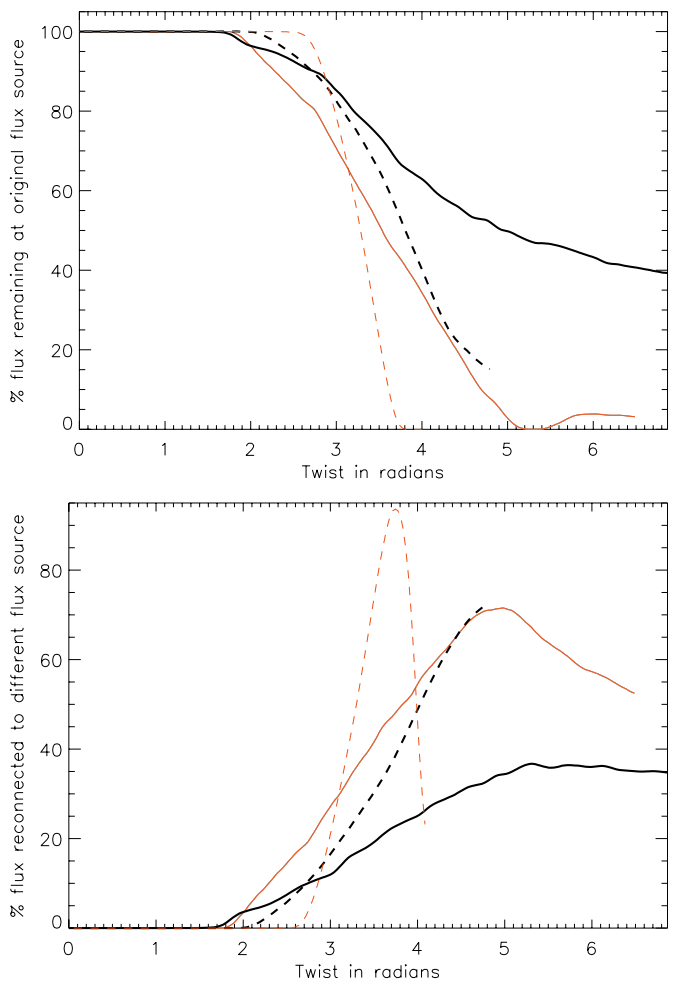

Fig. 11. The evolution of the flux (as a percentage of the total flux in the source) remaining at its original connection (top) and reconnected to the other source (bottom), as a function of the rotational/spinning angle. Solid and dashed lines represent the spinning and rotational experiments, respectively. Thick lines represent the $B_{\mathrm{bg}}=0$ case, thin lines $B_{\text {bg }}=0.05$.

that in the final image of the bottom row of Fig. 10 some pale blue field lines appear again. These are field lines that have reconnected again, and hence, have returned to their original connection. Indeed, at around $\theta \approx 5.2$ all the flux in the $B_{\text {bg }}=0.05$ case has changed connection (see also Table 1 ) with about $4 \%$ of the flux returning to its original connectivity by the end of the experiment.

Let us now compare the evolution of the footpoint connectivity for both the spinning and rotational driving of the flux concentrations. As "reconnection" is instantaneous in the potential approximation, the flux connectivity only depends on the relative alignment of the flux sources on the top and bottom boundaries. Hence, the spinning potential evolution is identical to the initial setup at all times, whereas the potential evolution is cyclical in nature when the sources are rotated on the boundaries, as the alignment changes cyclically. Figure 11 shows the dynamical evolution of the percentage of flux still remaining at its original connection (top) and flux which has reconnected to the other source on the top boundary (bottom), both as a function of the rotating (dashed lines) or the spinning (solid lines) angle. The $B_{\mathrm{bg}}=0$ experiments are represented by thick lines, the $B_{\text {bg }}=0.05$ ones with thin lines. The onset of reconnection occurs slightly later for the rotational driver, regardless of the presence of a background field. Once started, the reconnection rate appears fastest for the rotated experiment with an added background field and slowest for the spinning, no-background experiment. This is not entirely surprising, as it agrees roughly with the amount of energy that is injected as Poynting flux through the boundaries. The rotational driver affects a much larger fraction of the boundary and hence we expect the rotational experiments
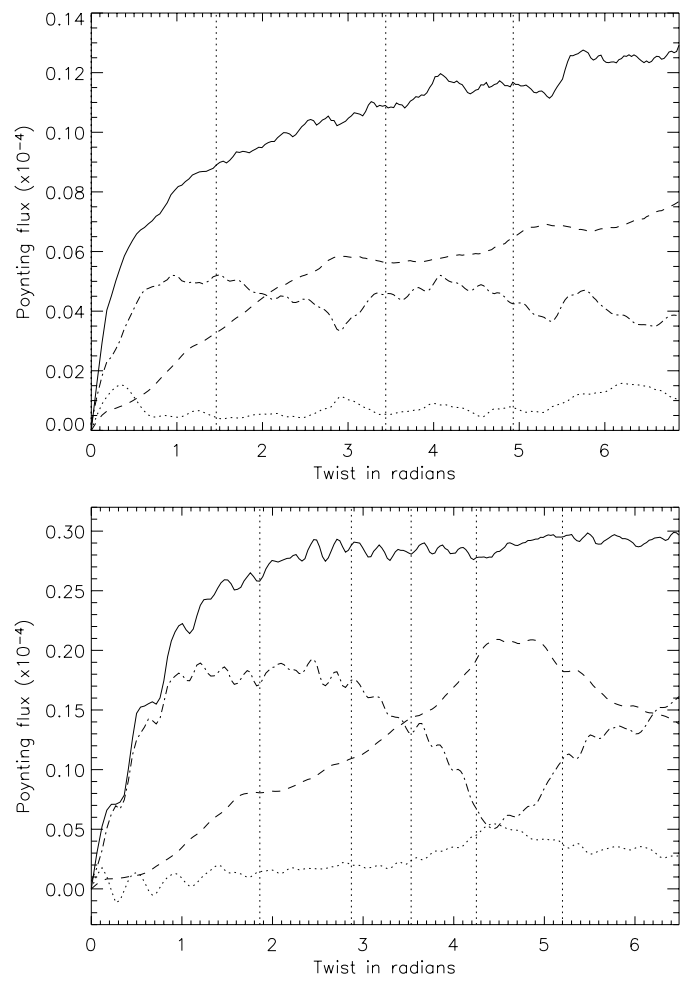

Fig. 12. The Poynting flux as a function of spinning angle, for the case $B_{\mathrm{bg}}=0($ top $)$ and $B_{\mathrm{bg}}=0.05$ (bottom). The dot-dashed line shows the change in magnetic energy, the dashed line is the joule dissipation and the dotted line represents the work done by the Lorentz force. The vertical dotted lines represent the angles at which reconnection starts, $25 \%$, $50 \%, 75 \%$ and $100 \%$, respectively, of the flux have reconnected in each case.

to be more energetic than their spinning counterparts. This is confirmed when comparing the rate at which the flux is reconnected (the thin - rotating - lines have a much steeper gradient than the thick - spinning - lines in Fig. 11) and at this stage, the rotated, background field case actually has the largest current in the central region of the domain (see Fig. 9). Although starting later for each of the drivers, the experiments with an added background field have the faster rate of reconnection for the same reason: the entire boundary is now permeated by magnetic field and hence, the boundary driving stresses a larger magnetic area, again resulting in a higher Poynting flux being injected into the system. Additionally, the confining effect of the background field results in a higher flux density as the flux tubes enter the central current sheet. Hence, the current magnitude will be higher (see also Fig. 9), resulting in a faster reconnection rate. Finally, we note that the spinning, $B_{\mathrm{bg}}=0.05$ case is the only of the four experiments where a small percentage of flux has returned to its original connectivity before the end of the numerical simulation. For the spinning $B_{\mathrm{bg}}=0$ case, the reconnected flux at the end of the experiment (i.e. after a twist of $\theta \approx 6.9$ ) only adds up to $61 \%$ of the total amount of flux in the source, implying that $39 \%$ (still decreasing with time) remains at its original connection.

\section{Energetics}

To provide some additional insight into the dynamical evolution, it is useful to study the energy flow in the system. The boundary driving results in a Poynting flux, injecting magnetic energy into the system. The Poynting flux, as a function of the spinning angle is plotted in Fig. 12 for both (top) $B_{\mathrm{bg}}=0$ and (bottom) 

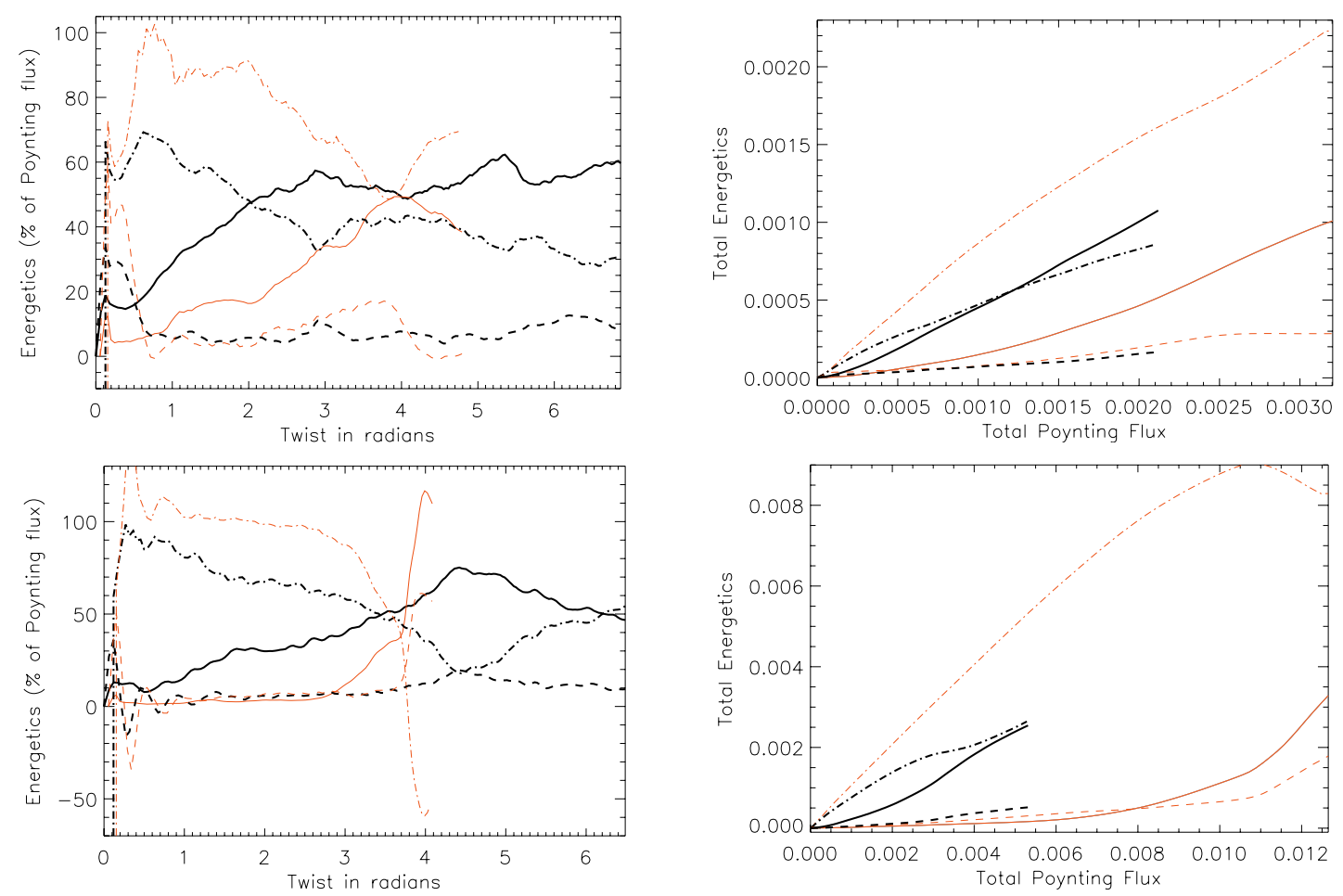

Fig. 13. (Left) The change in magnetic energy (dot-dashed lines), the Joule dissipation (solid lines) and the work done by the Lorentz force (dashed lines) as a function of the rotation (thin lines) or spinning (thick lines) angle, for both (top) $B_{\mathrm{bg}}=0$ and (bottom) $B_{\mathrm{bg}}=0.05$. (Right) The integrated volume contributions, as a function of the integrated Poynting flux. Top no-background field - Bottom constant background field.

$B_{\mathrm{bg}}=0.05$. The overall behaviour of the Poynting flux is very similar in both experiments but the magnitude of the Poynting flux is about twice as large when a background field is present, as the boundary driving affects a much stronger magnetic field, at large radii, in this case. The Poynting flux can be rewritten as three volume contributions to the change in magnetic energy density inside the 3D domain: the rate of change of the magnetic energy (dot-dashed line), the ohmic heating (dashed line) and the work done by the Lorentz force (dotted line). Qualitatively, there are many similarities between the two experiments: the boundary driving of the flux concentrations results in a constant increase in the magnetic energy. However, the rate of the build up of magnetic energy slows down at the onset of reconnection (first vertical, dotted line), when some of the stress imposed on the magnetic field is released. As current concentrations form, the Joule dissipation builds up, slowly at first, but more rapidly later on, when strong current concentrations are present in the domain. Eventually, the Joule dissipation becomes larger than the change in the magnetic energy. Throughout the experiments, the total magnetic energy continues to increase, indicating that neither of the systems have reached a steady state situation. The work done by the Lorentz force remains relatively low and only shows a small overall increase throughout the experiments. Finally, we note that after about $75 \%$ of the flux has changed connectivity (4th vertical, dotted line), the magnetic energy builds up faster again in the background field case, as the driver now causes the newly reconnected field to become increasingly stressed and tangled.

Qualitatively, the overall behaviour of the energy flow is the same for both types of footpoint driving, and a comparison is made in Fig. 13. The graphs on the left show the evolution of the three volume contributions to the Poynting flux, as a percentage of the Poynting flux, whereas the figures on the right show the same three contributions but now integrated, as a function of the integrated Poynting flux. The ohmic heating is represented by solid lines, the rate of change of the magnetic energy by dotdashed lines and the dashed lines show the work done by the Lorentz force. Thick and thin lines correspond to the spinning and rotating of the flux sources, respectively.

Let us firstly focus on the evolution of the energy contribution (as a percentage of the corresponding Poynting flux) simply as a function of the angle $\theta$ for the no-background case (Fig. 13, top left). The rate of change of magnetic energy (dot-dashed) constitutes a much larger fraction of the Poynting flux for the rotating experiments (thin lines), whereas the Joule dissipation (solid lines) very quickly becomes larger in the spinning experiments (thick lines). This can be understood from the different nature of the footpoint motions: the spinning of the flux concentrations causes a large shear at the centre of the domain and hence the central current sheet, through which reconnection between the flux tubes can take place, builds up very rapidly. Therefore, the Joule dissipation quickly becomes the dominant component. The rotational driving causes a much slower build up of the central current sheet and hence there is a much longer build up of magnetic energy before it is released through magnetic reconnection. It is only then that the Joule dissipation forms a larger contribution than the growth in magnetic energy in the rotational experiments. When a background field is present (Fig. 13, bottom left), the changeover of the relative importance of the change of magnetic energy and Joule dissipation does not seem to depend as strongly on the nature of the boundary motions (the thick and thin, solid and dot-dashed lines all cross at approximately the same value of $\theta$ ). However, there does seem to be a difference in the relative importance of the different components. In particular for the rotated, background field experiment, the vast majority of the Poynting flux seems to be in the rate of the change of the magnetic energy, with very little going into Joule dissipation (and the work done by the Lorentz force). Figure 13 
(right) shows the same three volume (integrated) contributions to the Poynting flux, but this time as a function of the integrated Poynting flux. Comparing the rate of change of the magnetic energy and the Joule dissipation, we see that for the rotating experiments, the former is always larger, as expected from the nature of the two types of boundary driving. This difference is most pronounced when a background field is added. For the spinning motions, the Joule dissipation and the rate of change of the magnetic energy appear to form comparable contributions.

In summary, for small values of the rotating/spinning angle (or a small amount of Poynting flux), a larger fraction of the injected energy is released as Joule dissipation in the spinning experiments. For the rotational footpoint driving, there is a much larger (and longer) build up of magnetic energy before it is released through ohmic dissipation. We will elaborate on the consequences of these differences in Sect. 7, where we compare the results of our 3D numerical simulations with the analytical study of Priest et al. (2005).

\section{Discussion}

The study presented in this paper, together with the results from Paper I, allows us now to compare the four different experiments, namely rotational and spinning boundary driving of two flux concentrations, both with and without an additional, constant background magnetic field. As pointed out several times, the global, dynamical evolution of the four cases shows many similarities: the boundary motions cause the magnetic field lines to become increasingly twisted and tangled and this shear in the magnetic field leads to the build up of a strong central current sheet. Once strong enough, this central current sheet allows reconnection to take place and the field lines gradually change connectivity. The Poynting flux injected through the boundaries of the domain leads to an increase of both the magnetic energy and the Joule dissipation (as well as a small amount of work done by the Lorentz force) inside the computational domain. Although the overall, dynamical evolution of the experiments shows many similarities, there are some important, underlying differences. These are found in particular in the structure of the magnetic field, in the shape and the growth rate of the central current sheet, the extent of the reconnection region at the centre of the domain and in the timing and efficiency of the reconnection between the two flux tubes.

The large-scale, rotational motions break the symmetrical alignment of the flux sources, leading to the build up of an increasingly twisted current sheet. The small-scale, spinning motions, on the other hand, preserve the initial alignment of the flux concentrations on the boundaries and hence, generate a straight current sheet at the centre of the domain. As the boundary driving continues, the Lorentz force generates a stagnation point flow of the field towards the central region, where the tension in the rotated field lines is the dominant component for the rotational motions and the magnetic pressure is the driving force of this process for the spinning of the flux sources. Once reconnection starts, the central current sheet bifurcates into a distinct, $x$-type configuration along the (quasi-)separatrix surfaces in the rotational experiments. This $x$-structure is compressed into an "elongated" $x$-type configuration (or two connecting $y$-points) for the spinning motions, due to the strong pressure in the inflow regions.

The effect of an additional background field seems largely the same, independent of the type of footpoint motion, namely that these experiments tend to be more energetic. Firstly, the added background field means that a higher amount of magnetic flux is present in the domain, regardless of the boundary driving. Secondly, the higher energies are a natural consequence of the fact that the boundary motions affect an area with a larger magnetic flux and hence, a higher Poynting flux is injected through the boundaries of the domain, leading to an increase in the free magnetic energy that is available for release. For exactly the same reason, the rotating experiments are more energetic than the spinning of the footpoints, as the boundary rotation affects a larger part of the domain (compare Figs. 1 in the current paper and Paper I).

When comparing the different types of footpoint motions, an obvious question to ask is which one is the most efficient. Using a reduced MHD model, Priest et al. (2005) investigated essentially the same experiments (although only for $B_{\mathrm{bg}}=0$ ) analytically, in order to compare the energy storage and heating that occurs at separatrix surfaces and separators. In their analysis, a separator current sheet forms due to large-scale, rotational motions and a separatrix current sheet due to small-scale spinning of the sources. These authors concluded that, in general, coronal heating is of comparable importance at separators and separatrices but in particular, that separatrix heating is more effective for small footpoint motions: the energy produced by spinning the flux concentrations exceeds that generated by rotating them by the same angle, for small values of the spinning and rotating angle, respectively. Although the distinction between "separator" and "separatrix" current sheets is no longer valid in the fully dynamical evolution in our numerical study, we can still compare the effect of large and small scale boundary motions. When looking at the build up of the maximum current in the central current sheet, the numerical simulations indeed find that the current builds up much more rapidly when the flux concentrations are spun on the boundaries (see Fig. 9). In other words, for small values of the angle $\theta$, a higher current density is reached for the spinning of the sources. However, for larger values of the rotating/spinning angle, the central current sheet becomes stronger for the rotational motions. Looking at the energetics, as a function of the rotating/spinning angle (Fig. 13, left), there is also a clear difference between the two types of boundary motions. Although the change in magnetic energy takes up a larger fraction of the Poynting flux for the rotational driving, relatively more energy is dissipated by the Joule dissipation for the spun footpoints. However, we have to keep in mind that for the same angle of spinning and rotating, less Poynting flux is injected into the domain for the spin driving. Hence, it is useful to also compare the amount of stored and released energy as a function of the total Poynting flux (Fig. 13, right). For the same amount of injected Poynting flux, more magnetic energy has built up for the rotational driving, but the Joule dissipation (and hence the heating) is higher for spinning of the footpoints. However, comparing the total Joule dissipation for both types of footpoint driving (thick and thin solid curves, Fig. 13, right), the difference is only about a factor of two, when $B_{\mathrm{bg}}=0$. Qualitatively, this agrees with the conclusions of Priest et al. (2005) that heating due to small scale (spinning) and large scale motions (rotating) are of a comparable magnitude. When a background field is present, however, there is more than an order of magnitude difference (factor of 15) between the amount of Joule dissipation in the spinning and rotating experiments. In this case, heating due to small scale footpoint motions seems considerably more efficient.

Although the experiments were designed specifically to investigate the effect of different types of footpoint motions, and not to model any particular solar event, putting our results into a solar context still provides some useful insights. The numerical 
Table 2. An overview of the time (in hours) at which various stages of the reconnection occur, for different values of the additional background magnetic field, $B_{\mathrm{bg}}$ and different types of footpoint motions.

\begin{tabular}{lcccc}
\hline \hline & \multicolumn{2}{c}{ Rotating } & \multicolumn{2}{c}{ Spinning } \\
\hline & $B_{\mathrm{bg}}=0$ & $B_{\mathrm{bg}}=0.05$ & $B_{\mathrm{bg}}=0$ & $B_{\mathrm{bg}}=0.05$ \\
\hline Onset of reconnection & 1.15 & 1.37 & 0.85 & 1.08 \\
25\% reconnected flux & 1.87 & 1.74 & 2.00 & 1.67 \\
$50 \%$ reconnected flux & 2.18 & 1.87 & 2.86 & 2.05 \\
75\% reconnected flux & 2.47 & 1.99 & - & 2.47 \\
$100 \%$ reconnected flux & - & 2.14 & - & 3.05 \\
End of experiment & 2.75 & 2.32 & 3.99 & 3.77 \\
\hline
\end{tabular}

simulations are done using a non-dimensional form of the MHD equations and dimensional quantities can be obtained by choosing three characteristic values and a set of scaling relations. In Paper I, these three characteristic values were chosen as a unit of magnetic field strength, $B_{0}=6 \mathrm{G}$, an electron number density, $n_{\mathrm{e}}=10^{15} \mathrm{~m}^{-3}$ and a unit of length, $l_{0}=77 \mathrm{Mm}$. Using these values, the initial numerical setup corresponds to:

$\rho_{(t=0)} \approx 1.7 \times 10^{-13} \mathrm{~kg} / \mathrm{m}^{3}$,

$T_{(t=0)} \approx 8.3 \times 10^{5} \mathrm{~K}$,

$p_{(t=0)} \approx 2 \times 10^{-3} \mathrm{~Pa}$,

$B_{\max (t=0)} \approx 5.4 \mathrm{G}$,

$B_{\mathrm{bg}(t=0)} \approx 0.3 \mathrm{G}$,

$v_{\text {driver }} \approx 9 \mathrm{~km} \mathrm{~s}^{-1}$,

$r_{0} \approx 5 \mathrm{Mm}$,

where $B_{\max }$ is the maximum magnetic field strength of the sources and $r_{0}$ is the radius of the sources. To avoid needless repetition, we refer the reader to the discussion section in Paper I for the derivation of the above values. Density, temperature and pressure correspond to typical (low) coronal values, whereas the size and field strength of the flux concentrations are in the range of X-ray bright points. The above set of initial values allows us to compare the timing of the various phases of the reconnection process in all four experiments. Table 2 provides an overview of when (in hours since the start of the footpoint motions) the different stages of the reconnection process occur. For example, the onset of reconnection takes place after $1.15 \mathrm{~h}$ when the flux concentrations $\left(B_{\mathrm{bg}}=0\right)$ are rotated on the boundaries, but after only $0.85 \mathrm{~h}$ for spinning motions. When a background field is present, the onset of reconnection goes up to 1.37 and $1.08 \mathrm{~h}$, for rotating and spinning of the footpoints, respectively. Comparing the two background field experiments, we see that $100 \%$ of the flux has changed connectivity after just over $3 \mathrm{~h}$ when the footpoints are spun, but after only about two thirds of that time $(2.14 \mathrm{~h})$ for the rotational footpoint driving.

Using the dimensional quantities and the information on the energy flow in the system from Fig. 13, we can now compare the energy that would be released by the reconnection process. Obviously, we have to keep in mind that the resulting values will depend strongly on our choice for the characteristic values, $B_{0}$, $n_{\mathrm{e}}$ and $l_{0}$. For the current choice, the free magnetic energy is plotted as a function of time (in hours) for the four different cases in Fig. 14 (top). Depending on the presence of a background field and the type of boundary driving, the amount of energy available for release reaches values in the range of $(1.26-5.37) \times 10^{27} \mathrm{erg}$. As we already know, the highest amount of free magnetic energy builds up in the rotated, background field experiment, in the least amount of time. A maximum of about $5.37 \times 10^{27} \mathrm{erg}$ is reached after only $2.2 \mathrm{~h}$. However, as the respective systems do not relax
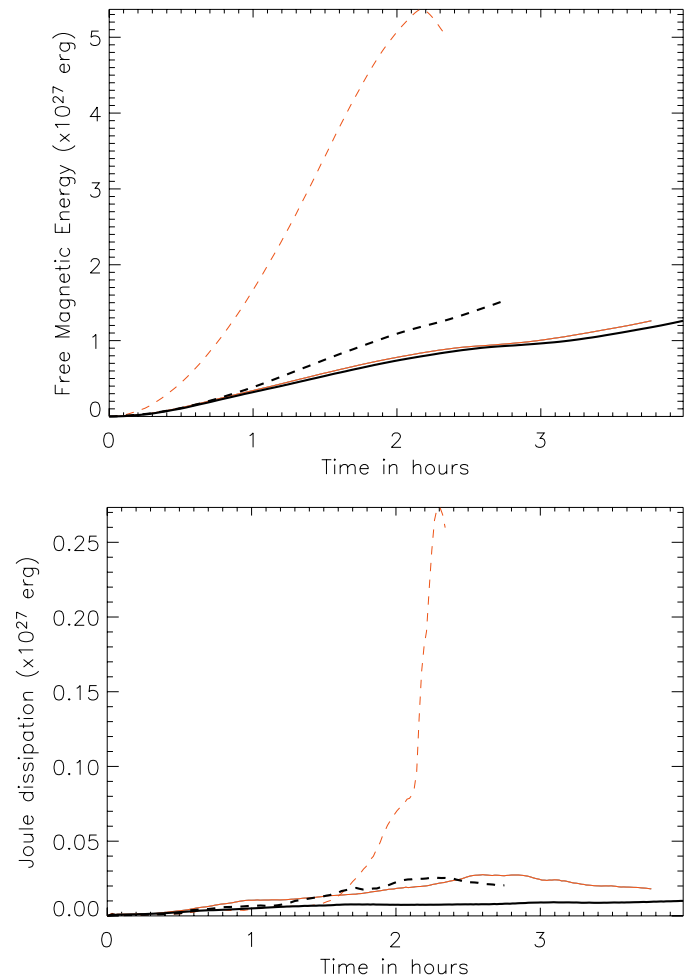

Fig. 14. (Top) The free magnetic energy and (bottom) Joule dissipation as a function of time where solid and dashed lines represent the spinning and rotational experiments, respectively. Thick lines represent the $B_{\mathrm{bg}}=$ 0 case, thin lines $B_{\mathrm{bg}}=0.05$.

to an equilibrium state, not all of this available energy is actually released. The amount of energy released through Joule dissipation is plotted in Fig. 14 (bottom), again as a function of the time in hours. The amount of Joule dissipation for the different experiments is in the $(0.026-0.27) \times 10^{27}$ erg range and again the highest values are reached at the end of the rotated, background field case. Integrated over time, the total amount of energy released by Joule dissipation is about $(0.51-1.47) \times 10^{27} \mathrm{erg}$, which is of the order of various small-scale heating events, such as X-ray bright points (Parnell 2002). However, we remind the reader again that the values quoted above depend strongly on the choice of the initial characteristic parameters, and additionally, that the amount of dissipated energy depends on the form of the resistivity and viscosity used in the numerical experiments (Mellor et al. 2005).

Finally, we point out that the experiments have some significant shortcomings, which were described in detail in Paper I. In particular, our model atmosphere has an initially constant pressure and hence, the resulting plasma $\beta$ values are much higher than expected in the solar corona. This could be addressed by the addition of gravitational stratification to the model, which would 
result in more realistic values of the plasma $\beta$ throughout the domain. Although this is likely to affect the reconnection outflow velocities, we do not expect it to cause significant changes in the global dynamical evolution of the various experiments. The periodic boundary conditions and the unrealistic, symmetrical alignment of the flux tubes could be seen as additional shortcomings of our model.

\section{Conclusions}

This paper firstly concentrates on a qualitative description of $3 \mathrm{D}$ numerical simulations of magnetic reconnection, driven by spinning of the footpoints of two adjacent flux tubes. The counter-spinning of the flux sources on opposing boundaries results in a very rapid build up of a separatrix current sheet at the central (quasi-)separatrix surface. This current sheet quickly becomes sufficiently strong to allow reconnection to take place, changing the connectivity of the footpoints.

The main aim of this paper is to compare the build up and subsequent release of magnetic energy due to two different types of boundary motions, namely rotating (see Paper I) and spinning of the flux concentrations. The main results of this comparison can be summarised as follows.

- The nature of the footpoint motions determines the structure of the magnetic field and the shape of the central current sheet; rotation of the sources generates a twisted current sheet, whereas spin motions lead to the build up of a straight current sheet.

- The spinning of the footpoints results in a higher shear in the central regions, leading to a faster build up of the amplitude of the current density and the quasi-separatrix layers (when $B_{\text {bg }} \neq 0$ ).

- The driving force behind the reconnection process is dominated by the tension and magnetic pressure for rotating and spinning, respectively.

- The spatial extent of the reconnection region is smaller in the rotational experiments, with reconnection mainly taking place near the central separator, where the currents are strongest. The separatrix current sheet in the spinning experiments has a relatively constant amplitude along its length, allowing reconnection to take place all along the current sheet.

- Although the onset of reconnection occurs sooner (at smaller angles) for spinning motions, the reconnection process is faster for rotational boundary driving.

- Generally, the rotational boundary motions and the addition of a constant background magnetic field lead to more energetic events, as more Poynting flux is injected through the boundaries.
- When no background field is present, the heating (i.e. the energy dissipated by Joule dissipation) due to large (rotating) and small scale (spinning) footpoint motions is comparable in magnitude, which qualitatively agrees with the conclusions of Priest et al. (2005). However, when a background field is added, heating due to small scale boundary motions (spinning) seems substantially more efficient (by an order of magnitude).

Acknowledgements. I.D.M. acknowledges support of a Royal Society University Research Fellowship. K.G. was supported by the Carlsberg Foundation in the form of a fellowship.

\section{References}

Aulanier, G., Pariat, E., \& Démoulin, P. 2005, A\&A, 444, 961

De Moortel, I., \& Galsgaard, K. 2006, A\&A, 451, 1101

Démoulin, P., Hénoux, J. C., Priest, E. R., \& Mandrini, C. H. 1996, A\&A, 308, 643

Démoulin, P. 2006, Adv. Space Res., 37, 1269

Falconer, D. A., Moore, R. L., Porter, J. G., \& Hathaway, D. H. 2003, ApJ, 593, 549

Galsgaard, K., \& Nordlund, Å. 1996, JGR, 101, 13445

Galsgaard, K., \& Nordlund, Å. 1997, JGR, 102, 231

Galsgaard, K., Parnell, C. E., \& Blaizot, J. 2000a, A\&A, 362, 395

Galsgaard, K., Priest, E. R., \& Nordlund, A. 2000b, Sol. Phys., 193, 1

Galsgaard, K., Titov, V. S., \& Neukirch, T. 2003, ApJ, 595, 506

Galsgaard, K., \& Parnell, C. E. 2005, A\&A, 439, 335

Green, R. M. 1965, in Solar and Stellar Magnetic Fields, ed. R. Lüst (Amsterdam: North Holland), IAU Symp., 22, 398

Gudiksen, B. V., \& Nordlund, A. 2005, ApJ, 618, 1031

Haynes, A., Parnell, C. E., Galsgaard, K., \& Priest, E. R. 2006, Proc. Roy. Soc., submitted

Klimchuck, J. A. 2006, Sol. Phys., 234, 41

Mellor, C., Gerrard, C. L., Galsgaard, K., Hood, A. W., \& Priest, E. R. 2005, Sol. Phys., 227, 39

Mikić, Z., Schnack, D. D., \& van Hoven, G. 1989, ApJ, 338, 1148

Milano, J. M., Dmitruk, P., Mandrini, C. H., Gómez, D. O., \& Démoulin, P. 1999, ApJ, 521, 889

Nordlund, A., \& Galsgaard, K. 1997, A 3D MHD code for Parallel Computers, Technical report, Astronomical Observatory, Copenhagen University

Parker, E. N. 1972, ApJ, 174, 499

Parnell, C. E. 2002, ESA-SP 505, 231

Parnell, C. E., \& Galsgaard, K. 2004, A\&A, 428, 595

Petschek, H. E. 1964, NASA Spec. Publ. SP-50, Physics of Solar Flares, ed. W. N. Hess, 425

Pevtsov, A. A., \& Acton, L. W. 2001, ApJ, 554, 416

Priest, E. R., \& Démoulin, P. 1995, J. Geophys. Res., 100 A12, 23443

Priest, E. R., Heyvaerts, J. F., \& Title, A. M. 2002, ApJ, 576, 533

Priest, E. R., Longcope, D. W., \& Heyvaerts, J. F. 2005, ApJ, 624, 1057

Syrovatskii, S. I. 1981, ARA\&A, 19, 163

Titov, V. S., Hornig, G., \& Démoulin, P. 2002, J. Geophy. Res., 107, A8, 3-1

Titov, V. S., Galsgaard, K., \& Neukirch, T. 2003, ApJ, 582, 1172

Van Ballegooijen 1986, ApJ, 311, 1001 